\title{
No Longer a Game of Chance: Playing the Cards of Genetics with Nutrition
}

\author{
Breann Abernathy, Erica Chung and Charles C Muscoplat
}

Department of Food Science and Nutrition, University of Minnesota, Minneapolis, USA

*Corresponding author: Charles C Muscoplat, McKnight Presidential Professor, Professor of Food Science and Nutrition. Department of Food Science and Nutrition, University of Minnesota, 717 Delaware St. SE. Room 478, Minneapolis, MN 55414, USA, Tel: 612-624-5387; Mobile: 612-590-0981; E-mail: cmuscop@umn.edu

Received date: 08 Sep, 2014, Accepted date: 24 Oct, 2014, published date: 31 Oct, 2014

Copyright: (c) 2014 Abernathy B, et al. This is an open-access article distributed under the terms of the Creative Commons Attribution License, which permits unrestricted use, distribution, and reproduction in any medium, provided the original author and source are credited.

\section{Short Communication}

In the days of James Cook and Christopher Columbus, humanity's greatest dietary triumph was realizing that eating citrus fruits such as limes, lemons and oranges, as well as onions cured what seafarers called "scurvy". Back in 1788 William Stark in England conducted a series of brilliant dietary experiments primarily on himself to better understand the sailors' findings of scurvy prevention by eating fresh citrus fruits. Stark ultimately died of scurvy as he was attempting to reproduce the disease in himself [1]. Nearly all vitamins were discovered by combination of serendipity, good luck and careful early scientific observation [2]. Thus, the study of nutrition was born. Fast forward several hundred years to present day, where nutritionists now know that scurvy is caused by a deficiency in vitamin C. The field of nutrition has come far since its beginnings, yet the field is still far from utilizing food in its highest form: personalized nutrition. Yes, there have been blood type diets, paleo diets, low and high fat diets, and diets of every type scattered in between, but the most ideal form of personalized nutrition stems from a field that is only now emerging from the shadows - epigenetics.

Epigenetics refers to the modulation of gene expression, due to a variety of environmental and lifestyle-related factors [3]. These changes do not alter the DNA sequence, but rather, how the genes are expressed, which means they can be regulated on or off and also up or down in their intensity. Epigenetics defies the long-standing Darwinian and Mendelian approach to genetics, and instead embraces a quasi- or pseudo-Lamarckian approach to inheritance, focusing on the concept that changes in a person's methylation pattern can induce profound long term personal changes and maybe, more importantly, transgenerational effects. Lamarck erroneously theorized about 'soft inheritance,' meaning that organisms grew the organs that they needed throughout life based on their environment, and those that were not used were not passed down transgenerationally. He even went as far as to say that giraffes lengthen their necks due changes in their environment (such as the height of the trees), which we now know to not be true. Lamarck also theorized that that environment gave rise to changes such as blindness in moles or teeth in mammals, rather than in Mendelian genetics or Darwinian evolution [4]. Contrastingly, modern epigenetics is real phenomenon, capable of producing dramatic biological changes in gene expression. The changes are brought about by chemical, dietary, environmental and even psychological factors, without any germ line DNA changes. The totality of these factors has been termed as the exposome, which is unique to each individual [5]. The merging of epigenetics with nutrition leads to an innovative field of study: nutrigenomics. What is nutrigenomics and what does it have to offer? Identical twins can be used as a prime model to explain the most fundamental and important notes of this field.
Nutrigenomics is the study of how nutrition and dietary constituents cause an alteration in gene expression, resulting in differing outcomes [3]. With the completion of the Human Genome Project, new technologies have come about to aid in the understanding of phenotypic outcomes, both dependent and independent of genotype. These new tools have been coined as the "omics" technologies, and provide high throughput that is useful in nutrigenomics research. The tools are comprised of genomics, transcriptomics, proteomics and metabolomics, and are combined with the dietary component of the exposome to better understand the complexities of nutritional genomics [6].

Monozygotic (MZ) twins are of interest here because they are born with identical genomes, as well as epigenomes. While their genomes are identical at birth, scientists have shown that a variety of environmental, lifestyle and nutritional factors can alter the epigenome; therefore altering the phenotypic outcome of individuals $[7,8]$. This discovery is most apparent in twins that live under vastly different conditions, and supports the observation that even though a person is given a permanent set of cards at birth, he or she may be able to alter the outcome by playing his or her hand of cards correctly.

For instance, in a set of MZ twins, one twin may choose to move to Alabama while the other twin may choose to move to Japan, where there are profound cultural differences in diet and lifestyle. This emigration can induce profound differences in each of the twins' health for the rest of their lives. Manifestation of cancer, diabetes and obesity are simply a few possibilities. For example, the use of green tea and the misuse of vitamin and antioxidant supplements can alter one's susceptibility to cancer, while vitamin D supplementation can alter insulin sensitivity. Although the above claims seem bold, they have been supported repeatedly through studies.

In recent years, the notion that drinking high quantities of green tea, similar to the average daily intake of individuals in Japan, can be beneficial to one's health, specifically in cancer prevetinon. This is due, in part, to the action of phytochemicals in green tea, specifically green tea polyphenols (GTP), acting on the epigenome of the individual that is consuming green tea [9]. When studying prostate cancer, it has been shown that a precursor is the hypermethylation at the glutathione-Stransferase pi (GSTP1) gene promoter [10]. GSTP1 is a metabolic enzyme that catalyzes the conjugation of a variety of toxins (specifically carcinogens), allowing them to be excreted [11]. A recent study showed that when a cell is exposed to GTP, DNA methyltransferase (DNMT, which adds methyl groups to DNA), is inhibited [12]. This causes the CpG regions of GSTP1 to be hypomethylated, allowing for the up-regulation of GSTP1. Since GSTP1 catalyzes the metabolism of carcinogens in the body, the upregulation of expression causes decreased carcinogen levels. This is a positive outcome for the twin living in Japan, where higher levels of green tea are consumed. Due to increased glutathione activity and 
metabolism of toxins, the twin is less likely to have prostate cancer later on. While this finding in genomic regulation is significant, prostate cancer is not the only cancer affected by green tea, nor is GTP the only class of phytochemicals that has such a chemopreventive effect.

It is widely recognized that some nutritionists and health professionals have been extensively promoting supplementation with vitamins and antioxidants to prevent the onset of acute and chronic diseases over the past decades. This has begun to change with further research. While antioxidants such as vitamins $\mathrm{A}, \mathrm{C}$ and $\mathrm{E}$ are all essential vitamins to the diet and beneficial to the body, there are adverse effects that come with over supplementation [13]. Antioxidants have a multitude of functions, such as protecting DNA and cellular organelles, neutralizing Reactive Oxygen Species (ROS) and promoting cellular proliferation. That being said, there must be a balance between antioxidant and ROS levels within the body in order to maintain a healthy system. One issue that occurs when oxidative status is out of balance caused by excessive consumption of antioxidants is the regulation of the p53 gene [14]. This gene codes for a tumor suppressing protein, also called p53 [14]. An over-abundance or supraphysiological level of antioxidants in the body causes the down-regulation of p53, resulting in cancerous and precancerous mutations in the DNA to go undetected and unregulated. This leads to proliferation of carcinoma cells, and therefore increased tumor growth [14]. The promotion of cancer cell growth has been shown to be correlated with antioxidants in a variety of studies, focusing on both humans and small laboratory animals [15-18]. Notably, Martin Bergo et al, found that mice fed higher, 'supraphysiological' doses of vitamin $\mathrm{E}$ (similar in amount or ratio to many over-the-counter, health-store recommended human supplements), died twice as fast as the controls and had significant increases in lung tumor size and aggression [16]. Such studies show that patients that are diagnosed with chronic disease are not the best people to be taking antioxidant supplements, as it could be detrimental to their health. Oddly enough, these are the people that antioxidants are most commonly recommended to. By fully understanding the epigenetic changes that are brought on by such compounds, nutritionists and medical professionals alike can better assess the current and future recommendations for both healthy and unhealthy individuals.

Another worldwide mystery of nutrition is diabetes mellitus and the multiple dispositions that influence the manifestation of type I or II diabetes. Several recent studies have shown that correcting vitamin D deficiency induces insulin sensitivity and assists in the control of diabetes in both obese children and adults [19]. A 2014 study found that vitamin D deficiency was most prominent in obese children and when that deficit was corrected, inflammation of the body and inadequate insulin response was ameliorated [19]. A similar study conducted in 2013 focused on obese adults that were supplemented with 4000 IU of vitamin D3 per day. Researchers determined that treating vitamin $\mathrm{D}$ deficiency was an effective addition to traditional treatments of diabetes [20]. This discovery carries many implications, especially for pre-diabetic and patients deficient in vitamin D. Additional supplementation could potentially eliminate or greatly reduce the manifestation of full-fledged diabetes in these patients and mitigate complications associated with the disease, which afflicts more than 25 million Americans, double that number worldwide and a dramatic increase in the developing world [21]. For the twins living in Japan and Alabama, this information could encourage the addition of vitamin D supplements to their regular diets if pre-diabetic symptoms manifest and would not require more powerful diabetic medications.
Perhaps one of the most stunning findings that scientists have been able to gather increasing evidence for is the environmental and nutritional factors not only affect the exposed persons, but also their future generations. Transgenerational obesity has been readily studied in the past few years and it has been determined that both maternal and paternal weight and nutrition influences the manifestation of obesity in their children. Paternal obesity is associated with hypomethylation of the IGF2, insulin growth factor 2 , site in newborns increasing their risk for contracting cancer and becoming obese in the future [22]. Maternal obesity or a late gestational high-fat diet increases the risk of metabolic diseases in the progeny [23,24]. For the twins, the one residing in Alabama is constantly exposed to the classic "Southern" diet, which will cause an increased risk of passing obesity to their children. In comparison, the twin living in Japan is highly likely to be consuming a significantly more healthy Japan diet, and is less likely to pass undesirable methylation patterns on to their children. Modifications in both the mother and father's diet and lifestyle pre-pregnancy may be the difference between a child becoming obese or contracting type I diabetes.

While diabetes and obesity is a now emerging problem, nutrigenomics has also been utilized in America to ameliorate Neural Tube Defects (NTD) in newborn for the past few decades [25]. U.S. Government food policy encouraging the supplementation of folic acid for pregnant females and also in certain food products has decreased the prevalence of NTD in America. Just as vitamins and minerals have been added to food over the 20th century, we may find other critical nutrients in the 21st century. However, worldwide, $300,000-400,000$ children are born each year with NTD, indicating that it is still a major problem. One of the challenges relates to how to translate these new dietary research findings to the rest of the world and specifically the developing world [25].

If food can impact our physical appearance and mental capabilities, can it alter our senses? Surprisingly, it can. The phrase "smelling fear" has never been taken literally until now. Studies have shown that humans and animals alike can be conditioned to elicit a fearful response to an action or item when it is coupled with negative stimuli. In addition, a 2013 study conducted by Brian Dias and his team at Emory University discovered that this fear could also be transgenerational. The offspring of male mice that had been conditioned to fear a cherry blossom like scent reacted more negatively towards that smell and also required lower dosages of that smell compared to normal mice illustrating an increased sensitivity to the smell of cherry blossoms. These studies were only performed using male mice and should not be generalized beyond male mice at this time [26].

Our knowledge of nutrigenomics is only now emerging. The use of "omics" technologies provides a rapidly growing wealth of insight into the interactions between the genome and the exposome. As we continue to learn, we can better identify the proper recommendations for each individual. It would be wrong to assign blame to either men or women on the potential inheritance of adverse traits due to dietinduced epigenetic changes. Society has brought about the current obesity epidemic via a multitude of factors such as cheap food, disposable income in developed and developing countries, modern agricultural productivity and governmental food policy. The reversal of the obesity epidemic will require addressing each of these alone and together along with these new, scientific tools.

While only a few examples have been discussed, diet-related epigenetics and nutrigenomics are key players in perfecting 
personalized nutrition and ameliorating or preventing acute and chronic diseases. However, many of these societal problems such as obesity, heart disease and cancer cannot be reduced or eliminated in the near term, or even near decades. Treating diet-related epigeneticrelated diseases is a multi-generational effort, and will be a long time in the making. Starting at the root of the problem and working towards the trees and branches will provide the knowledge and tools to begin to tackle this problem. Do not despair if you have been given a poor hand of cards to play in your DNA. Despite having some genetic predispositions to cancer, diabetes or obesity, like the set of twin's we have discussed, you may be able to alter your original outcome and perhaps prevent these diseases from manifesting or at least mitigate their impact on you. The new science of nutrigenomics may be one of the easiest and soonest manifestations of the new field of personalized medicine. Some trends in healthy food and healthy living is leading the way to personalized nutrition, wellness and reducing the burden of chronic disease in the US and worldwide.

\section{References}

1. Stark W (1780) The works of the late William Stark, M.D., consisting of clinical and anatomical observations, with experiments, dietetical and statical, revised and published from his original mss. by James Carmichael Smyth. London: J. Johnson.

2. Semba RD (2012) The discovery of the vitamins. Int J Vitam Nutr Res 82 310-315.

3. Kussmann M, Krause L, Siffert W (2010) Nutrigenomics: where are we with genetic and epigenetic markers for disposition and susceptibility? Nutr Rev 68 Suppl 1: S38-47.

4. Smythies J, Edelstein L, Ramachandran V (2014) Molecular mechanisms for the inheritance of acquired characteristics-exosomes, microRNA shuttling, fear and stress: Lamarck resurrected? Front Genet. doi:10.3389/ fgene.2014.00133. 5:133.

5. Miller GW, Jones DP (2014) The nature of nurture: refining the definition of the exposome. Toxicol Sci 137: 1-2.

6. Wittwer J, Rubio-Aliaga I, Hoeft B, Bendik I, Weber P et.al. (2011) Nutrigenomics in human intervention studies: current status, lessons learned and future perspectives. Mol Nutr Food Res. doi:10.1002/mnfr. 201000512. 55: 341-358

7. Wong AH, Gottesman II, Petronis A (2005) Phenotypic differences in genetically identical organisms: the epigenetic perspective. Hum Mol Genet 14 Spec No 1: R11-18.

8. Fraga MF, Ballestar E, Paz MF, Ropero S, Setien F, et al. (2005) Epigenetic differences arise during the lifetime of monozygotic twins. Proc Natl Acad Sci U S A 102: 10604-10609.

9. Li MJ, Yin YC, Wang J, Jiang YF (2014) Green tea compounds in breast cancer prevention and treatment. World J Clin Oncol 5: 520-528.

10. Lin X, Tascilar M, Lee WH, Vles WJ, Lee BH, et al. (2001) GSTP1 CpG island hypermethylation is responsible for the absence of GSTP1 expression in human prostate cancer cells. Am J Pathol 159: 1815-1826.
11. Henderson CJ, McLaren AW, Moffat GJ, Bacon EJ, Wolf CR (1998) Piclass glutathione S-transferase: regulation and function. Chem Biol Interact 111-112: 69-82.

12. Pandey M, Shukla S, Gupta S (2010) Promoter demethylation and chromatin remodeling by green tea polyphenols leads to re-expression of GSTP1 in human prostate cancer cells. Int J Cancer 126: 2520-2533.

13. Lobo V, Patil A, Phatak A, Chandra N (2010) Free radicals, antioxidants and functional foods: Impact on human health. Pharmacogn Rev 4: 118-126.

14. Thompson D (2014) Could Antioxidants Speed Up Cancer Progression? WebMD News. 1-3.

15. Pearson P, Lewis S a, Britton J, Young IS, Fogarty A (2006) the prooxidant activity of high-dose vitamin $\mathrm{E}$ supplements in vivo. BioDrugs. 20: $271-3$.

16. Ledford H (2014) Antioxidants speed cancer in mice. Nat News Comment. 1-3. doi:10.1038/nature.2014.14606.

17. Bjelakovic G, Nikolova D, Gluud LL, Simonetti RG, Gluud C (2007) Mortality in randomized trials of antioxidant supplements for primary and secondary prevention: systematic review and meta-analysis. JAMA 297: 842-857.

18. Baumann LS, Spencer J (1999) The effects of topical vitamin E on the cosmetic appearance of scars. Dermatol Surg. 25:311-315.

19. Belenchia AM, Tosh AK, Hillman LS, Peterson CA (2013) Correcting vitamin $\mathrm{D}$ insufficiency improves insulin sensitivity in obese adolescents: a randomized controlled trial. Am J Clin Nutr 97: 774-781.

20. Reyman M, Verrijn Stuart AA, van Summeren M3, Rakhshandehroo M4, Nuboer R5, et al. (2014) Vitamin D deficiency in childhood obesity is associated with high levels of circulating inflammatory mediators, and low insulin sensitivity. Int J Obes (Lond) 38: 46-52.

21. Guariguata L, Whiting DR, Hambleton I, Beagley J, Linnenkamp U, et al. (2014) Global estimates of diabetes prevalence for 2013 and projections for 2035. Diabetes Res Clin Pract 103: 137-149.

22. Soubry A, Schildkraut JM, Murtha A, Wang F, Huang Z, et al. (2013) Paternal obesity is associated with IGF2 hypomethylation in newborns: results from a Newborn Epigenetics Study (NEST) cohort. BMC Med 11: 29.

23. Attig L, Vigé A, Gabory A, Karimi M, Beauger A, et al. (2013) Dietary alleviation of maternal obesity and diabetes: increased resistance to dietinduced obesity transcriptional and epigenetic signatures. PLoS One 8: e66816.

24. Khalyfa A, Carreras A, Hakim F, Cunningham JM, Wang Y, et al. (2013) Effects of late gestational high-fat diet on body weight, metabolic regulation and adipokine expression in offspring. Int J Obes (Lond) 37: 1481-1489.

25. Green NS (2002) Folic acid supplementation and prevention of birth defects. J Nutr 132: 2356S-2360S.

26. Geddes L (2013) Fear of a smell can be passed down several generations. New Sci. doi:10.1016/S0262-4079(13)62827-4: 220:10. 